\title{
Saving the esophagus: At what cost?
}

\author{
Ross M. Bremner, MD, PhD
}

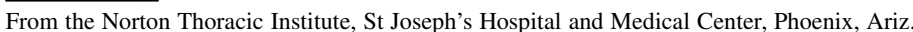

Disclosures: Author has nothing to disclose with regard to commercial support.

Received for publication Feb 28, 2018; accepted for publication March 6, 2018; available ahead of print Apr 13, 2018.

Address for reprints: Ross M. Bremner, MD, PhD, Norton Thoracic Institute, St Joseph's Hospital and Medical

Center, 500 W Thomas Rd, Suite 500, Phoenix, AZ 85013 (E-mail: Ross.Bremner@ DignityHealth.org).

J Thorac Cardiovasc Surg 2018;156:416-7

0022-5223/\$36.00

Copyright (c) 2018 Published by Elsevier Inc. on behalf of The American Association for Thoracic Surgery

https://doi.org/10.1016/j.jtcvs.2018.03.013

It was not that long ago that esophagectomy was the recommended treatment for high-grade esophageal dysplasia. Esophagectomy was almost always curative, but it was associated with increased morbidity (which remains high today, despite surgical improvements) and challenging long-term issues in quality of life. Interestingly, half of the pathologic specimens of patients who underwent esophagectomy for high-grade esophageal dysplasia revealed an invasive cancer, although it was usually still intramucosal (ie, T1a). Endoscopic treatments such as radiofrequency ablation (RFA) and cryotherapy quickly replaced esophagectomy as the first-line treatment, and these therapies can be combined with the suction-snare techniques of endoscopic mucosal resection (EMR) for intramucosal cancerous lesions (eg, T1a). Understandably, quality of life with one's original esophagus is superior to quality of life with a neoesophagus created from tubularized stomach or from colon, and local therapies such as EMR and RFA have greatly advanced the therapeutic options for patients with high-grade esophageal dysplasia.

The lamina propria is barren of lymphatics, and nodal disease is very unusual when the tumor is confined to the mucosa (ie, superficial to the muscularis mucosa). The submucosa is richly traversed by lymphatic vessels (Figure 1), however, and the incidence of nodal disease becomes much higher when the tumor invades through the muscularis mucosa. The timing of this invasion and ways to determine whether it has occurred remain unclear, but it is certain that local endoscopic therapies are ineffective once nodal involvement is present.

In their article in this this issue of the Journal, Nelson and colleagues ${ }^{1}$ have attempted to provide some guidance on how to proceed when a submucosal, clinically nodenegative esophageal cancer is encountered. This situation is uncommon, but it is encountered in high-volume centers, especially those with surveillance programs for Barrett's esophagus and those capable of safely performing aggressive EMR. The study includes a larger number of patients than reported in previous publications, and it includes patients treated conservatively after confirmation of submucosal invasion on EMR. (The dictum has been to perform

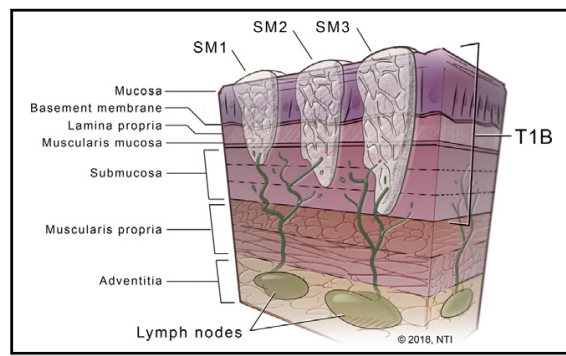

T1B subclass tumors encountering lymphatic tissue in submucosal layer of esophageal wall.

Central Message

Esophagectomy may not be necessary for small invasive cancers, or for well-differentiated or moderately differentiated cancers without lymphovascular invasion but with submucosal invasion only.

See Article page 406

esophagectomy when T1b occurs.) Nelson and colleagues ${ }^{1}$ have compared outcomes of patients treated with EMR alone against outcomes of patients treated with esophagectomy (with or without previous EMR). They have attempted to provide a scoring system to help practitioners stratify patients as being at low risk, intermediate risk, and high risk for relapse or for failure of local therapy. Nelson and colleagues ${ }^{1}$ are to be congratulated on their attempts to help clarify optimal therapy in this difficult subgroup of patients, and they deserve recognition for their excellent results with both surgical and endoscopic therapy.

There are some very interesting findings in this study. First, Nelson and colleagues ${ }^{1}$ had a $0 \% 30$-day mortality in their esophagectomy group — and if one is considering implementing a practice change in response to this article, one's own center should have similar excellent surgical outcomes. Second, of those patients who underwent esophagectomy after EMR, 50\% had no residual disease, implying that the surgery was unnecessary in this group of patients. We need a better way to predict this situation in the future, and new techniques of confocal endomicroscopy and volume laser endomicroscopy may help in this regard. In the study by Nelson and colleagues, ${ }^{1}$ local recurrence was seen in the conservatively treated group; in all 5 cases, however, the disease happened to be amenable either to further local therapy or to surgery. Interestingly, 3 of 5 patients with local recurrences had not been treated with RFA after EMR — an indication that RFA is an important adjunct to EMR. 


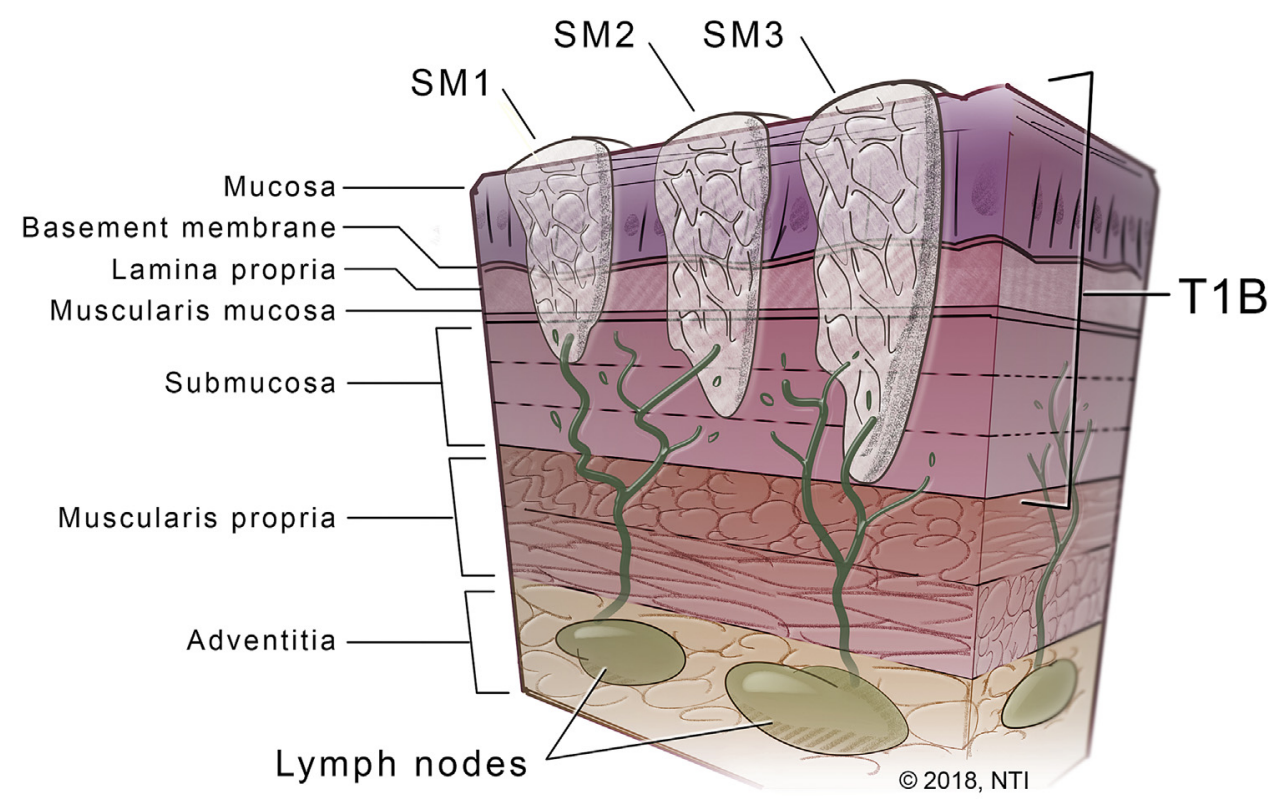

FIGURE 1. Illustration depicting T1B subclass tumors encountering lymphatic tissue in the submucosal layer of the esophageal wall. (Used with permission of Norton Thoracic Institute, Phoenix, Ariz.)

One thing does appear fairly clear. Esophagectomy may not be necessary for small invasive cancers, or for welldifferentiated or moderately differentiated cancers without lymphovascular invasion but with submucosal invasion only (preferably SM1, cancers limited to the superficial submucosa). Clear margins with EMR and subsequent RFA should be sufficient, but close follow-up is still needed. These patients should be treated in high-volume centers, where surgeons are comfortable performing interventional esophageal endoscopy and esophagectomy. Many patients' esophagi may be saved without high-risk surgery, with excellent long-term swallowing function. My fear, however, is that many patients with submucosal invasion who would have a very good chance at cure with esophagectomy may fall prey to the lure of noninvasive therapy and therefore receive inadequate treatment of an otherwise curable cancer. We certainly need further studies to let us know when it is safe to treat submucosal invasion with endoscopic therapy alone.

We are close, but we are not quite there yet in determining when an esophagus can reliably be saved and when it will need removal. The findings presented by Nelson and colleagues ${ }^{1}$ are useful for those of us weighing the risk of recurrence with the risk of surgery in this difficult cohort of patients with submucosal and clinically node-negative disease. My hope is that molecular analysis of these tumors will facilitate prediction of aggressiveness, and that this factor will be added to the scoring system.

\section{Reference}

1. Nelson DB, Dhupar R, Katkhuda R, Correa A, Goltsov A, Maru D, et al. Outcomes after endoscopic mucosal resection or esophagectomy for submucosal esophageal adenocarcinoma. J Thorac Cardiovasc Surg. 2018;156:406-13. 